\title{
Reduced Visual Acuity in Both Eyes of Monocularly Deprived Kittens Following a Short or Long Period of Reverse Occlusion
}

\author{
Kathryn M. Murphy and Donald E. Mitchell \\ Department of Psychology, Dalhousie University, Halifax, NS B3H 4J1, Canada
}

\begin{abstract}
While there can be substantial recovery of vision during reverse occlusion in the deprived eye of kittens that have been monocularly deprived from birth, in many situations this visual improvement does not appear to be retained following the introduction of binocular visual input. This study examines the consequences of periods of reverse occlusion whose onset and duration would be thought to maximize the opportunity for good and permanent recovery of vision in the initially deprived eye. Twenty kittens were monocularly deprived from near birth to either 4,5 , or 6 weeks of age and then reverse occluded for periods that were either very brief (9-18 d) or very long (9-12 weeks). In the former situation, binocular visual experience was introduced when the visual cortex was most susceptible to environmental modification, which would be thought to maximize the opportunity for the eventual development of good vision in both eyes. On the other hand, the long periods of reverse occlusion might be expected to lead to a permanent recovery of vision in only the initially deprived eye, since binocular visual input would have been introduced at a time when the cortex was no longer very plastic. Surprisingly, the end result of both these extreme regimens of reverse occlusion was the same, namely, a severe bilateral amblyopia in which the acuity of both eyes was permanently reduced to levels that were as low as $1 / 10$, but more typically between $1 / 3$ and $1 / 4$ of normal values. Contrast sensitivity functions measured on 2 of the cats that received a brief period of reverse occlusion revealed deficits in contrast sensitivity of about an order of magnitude at all spatial frequencies. These observations have important implications for the nature of the physiological and anatomical changes that occur in the visual cortex during reverse occlusion.
\end{abstract}

It is now well established that the developing visual system of many species of frontal eyed mammals is influenced profoundly by the nature of its early input (Movshon and Van Sluyters, 1981; Mitchell and Timney, 1984). Much of our current understanding of the role that early visual experience plays in development has been derived from studies of the remarkable

Received Sept. 4, 1986; revised Dec. 1, 1986; accepted Dec. 3, 1986.

This work was supported by Grant A7660 to D.E.M. from the Natural Science and Engineering Research Council of Canada. K.M.M. held the Ross C. Purse Fellowship from the Canadian National Institute For the Blind. We wish to thank Heather Dzioba and Jane-Anne Horne for their assistance with the measurements of visual acuity and for their dedicated care of the cats.

Correspondence should be addressed to Dr. K. M. Murphy, University of California, School of Optometry, Berkeley, CA 94720.

Copyright (C) 1987 Society for Neuroscience $0270-6474 / 87 / 051526-11 \$ 02.00 / 0$ consequences of an extreme form of biased visual exposure, namely, monocular deprivation. Following a period of monocular deprivation imposed within a certain sensitive period that extends to about 8 to 10 months of age in kittens and somewhat longer in monkeys (beyond 1 year), the overwhelming majority of cells in visual cortical area 17 can be excited only by stimuli presented to the nondeprived eye (Hubel and Wiesel, 1970; Cynader et al., 1980; LeVay et al., 1980; Jones et al., 1984). Although these effects of monocular deprivation are not so profound in layer IV as in the other cortical layers, nevertheless severe anatomical changes are evident in the pattern of geniculate afferents to this layer (Hubel et al., 1977; LeVay et al., 1980; Swindale et al., 1981). Afferents subserving the deprived eye shrink in extent, while those originating from the nondeprived eye expand to occupy territory that would normally be innervated by the deprived eye. Anatomical effects of comparable magnitude are also observed in the lateral geniculate nucleus (LGN) (Wiesel and Hubel, 1963; Guillery and Stelzner, 1970; Guillery, 1972). These various anatomical and physiological changes are accompanied by a severe reduction in the vision of the deprived eye, which can be rendered blind for a time following a period of early monocular deprivation (Giffin and Mitchell, 1978).

The substantial anatomical, physiological, and behavioral effects of monocular deprivation are not necessarily irreversible; considerable recovery is possible if normal visual input is restored to the deprived eye while the animal is still young. In cats some visual as well as physiological recovery occurs on simply restoring normal patterned visual input to the deprived eye (Mitchell et al., 1977a; Giffin and Mitchell, 1978). However, in order to promote visual recovery in monkeys it appears necessary to occlude the nondeprived eye at the time that visual input is restored to the deprived eyc, a proccdurc rcferred to as reverse occlusion (Blakemore et al., 1978; LeVay et al., 1980; Blakemore et al., 1981). This procedure, which forces the animal to use its initially deprived eye, also promotes somewhat greater behavioral and physiological recovery in kittens than that which occurs when the animal receives binocular visual exposure during recovery (Mitchell et al., 1977a). The physiological recovery promoted by reverse occlusion is very rapid in both cats and monkeys (Movshon, 1976; Blakemore et al., 1981). In kittens monocularly deprived to 4 weeks of age, the distribution of cortical ocular dominance in the visual cortex can be switched from complete dominance by the initially nondeprived eye, to dominance by the initially deprived eye after only $9 \mathrm{~d}$ of reverse occlusion (Movshon, 1976). Although the changes of ocular dominance are not quite so extreme in kittens reverse occluded at 5 or 6 weeks of age, the shifts of ocular dominance toward 
the initially deprived eye is virtually complete in only $18 \mathrm{~d}$ (Movshon, 1976). These rapid changes in cortical ocular dominance toward the initially deprived eye are accompanied by an equally rapid improvement in the vision of this eye (Giffin and Mitchell, 1978).

However, in an earlier investigation (Mitchell et al., 1984a, b), we showed that the substantial visual recovery promoted by reverse occlusion was not permanent. In most situations the vision of the initially deprived eye deteriorated rapidly following restoration of normal visual input to the other eye. The decline in the vision of the initially deprived eye following termination of reverse occlusion was accompanied by a gradual improvement in the vision of the initially nondeprived eye from the level to which it had been depressed by the period of reverse occlusion. However, although the vision of the initially nondeprived eye improved significantly in many of the conditions studied, the acuity of this eye never recovered to normal adult levels.

In an effort both to study these consequences of reverse occlusion further and to search for rearing conditions that might promote a better final outcome, we have explored the consequences of 2 extreme conditions of reverse occlusion that might be thought to optimize the possibility for good and permanent recovery of the vision of the initially deprived eye. The experiments were performed on kittens that were monocularly deprived from near birth until either 4,5 , or 6 weeks of age and that were then reverse occluded for either brief periods of time ( 3 weeks or less), in which case binocular visual input was introduced at a time when the visual system was still highly susceptible to environmental modification, or else much longer periods (9-12 weeks), so that the animals were much older when reverse occlusion was terminated. The short period of reverse occlusion employed in the first situation has been shown to be sufficiently long to ensure the maximum shift of cortical ocular dominance that is possible following the lengths of monocular deprivation used in this study (Movshon, 1976). Since binocular visual input would be introduced to these kittens at the time when cortical plasticity was close to its peak, we anticipated that this would maximize the opportunity for the eventual development of good vision in both eyes. At the other extreme, long periods of reverse occlusion would be thought to maximize the opportunity for long-lasting improvement in the vision of the initially deprived eye (while at the same time reducing the opportunity for substantial improvement in the vision of the other eye), since binocular visual input would be introduced toward the end of the sensitive period. Surprisingly, our findings indicate that neither of these regimens of reverse occlusion produced these expected outcomes.

\section{Materials and Methods}

\section{Animals and rearing conditions}

A total of 20 kittens, born and raised in a closed colony, were employed in this study. The eyelids of 1 eye of each kitten were sutured closed at about the time of natural eyelid opening (6-11 d of age) until the kittens were either 4,5 , or 6 weeks old. Eyelid closure was performed under gaseous halothane anesthesia supplemented by injections of a longlasting local anesthetic (Marcaine $0.25 \%$ ) around the palpebral aperture. The palpebral conjunctiva was first dissected free from both lid margins to expose about $3 \mathrm{~mm}$ of the eyelid tissue. The palpebral conjunctivae freed from the upper and lower lids were sutured together with 5-0 chromic gut. Following application of a broad-spectrum ophthalmic antibiotic (Chloromycetin 1\%), the exposed tissue on the underside of the eyelids was opposed and sutured together with 4-0 silk. Although
Table 1. Rearing histories and final acuities

\begin{tabular}{|c|c|c|c|c|}
\hline \multirow[b]{3}{*}{ Cat } & \multirow{2}{*}{\multicolumn{2}{|c|}{ Reverse occlusion }} & \multicolumn{2}{|c|}{$\begin{array}{l}\text { Eventual visual acuity } \\
\text { (cycles/deg) }\end{array}$} \\
\hline & & & \multirow{2}{*}{$\begin{array}{l}\text { Deprived } \\
\text { eye }\end{array}$} & \multirow{2}{*}{$\begin{array}{l}\text { Non- } \\
\text { deprived } \\
\text { eye }\end{array}$} \\
\hline & Imposed at & Duration & & \\
\hline $\mathrm{C} 168$ & 4 wk & $9 d$ & 1.64 & 1.91 \\
\hline $\mathrm{C} 276$ & $4 \mathrm{wk}$ & $9 \mathrm{~d}$ & 1.80 & 1.95 \\
\hline $\mathrm{C} 279$ & 4 wk & $9 \mathrm{~d}$ & 1.24 & 1.48 \\
\hline C294 & 4 wk & $9 \mathrm{~d}$ & 1.67 & 2.02 \\
\hline C170 & $4 \mathrm{wk}$ & $2 \mathrm{wk}$ & 0.62 & 2.31 \\
\hline $\mathrm{C} 181$ & 4 wk & 9 wk & 1.89 & 1.30 \\
\hline C199 & $4 \mathrm{wk}$ & $12 \mathrm{wk}$ & 2.55 & 1.25 \\
\hline C302 & $4 \mathrm{wk}$ & 12 wk & 2.78 & 0.80 \\
\hline $\mathrm{C} 159$ & $5 \mathrm{wk}$ & $9 \mathrm{~d}$ & 1.44 & 1.93 \\
\hline $\mathrm{C} 166$ & $5 \mathrm{wk}$ & $9 \mathrm{~d}$ & 1.91 & 2.42 \\
\hline $\mathrm{C} 155$ & $5 \mathrm{wk}$ & $18 \mathrm{~d}$ & 1.73 & 3.37 \\
\hline $\mathrm{C} 164$ & $5 \mathrm{wk}$ & $18 \mathrm{~d}$ & 2.62 & 2.62 \\
\hline $\mathrm{C} 142$ & $5 \mathrm{wk}$ & 9 wk & 1.88 & 3.58 \\
\hline $\mathrm{C} 157$ & 5 wk & $12 \mathrm{wk}$ & 2.20 & 1.25 \\
\hline $\mathrm{C} 295$ & $5 \mathrm{wk}$ & $12 \mathrm{wk}$ & 2.00 & 0.90 \\
\hline C298 & $5 \mathrm{wk}$ & $12 \mathrm{wk}$ & 2.75 & 1.20 \\
\hline $\mathrm{C} 262$ & $6 \mathrm{wk}$ & $18 \mathrm{~d}$ & 2.85 & 4.25 \\
\hline $\mathrm{C} 265$ & $6 \mathrm{wk}$ & $18 \mathrm{~d}$ & 3.08 & 3.60 \\
\hline C269 & $6 \mathrm{wk}$ & $18 \mathrm{~d}$ & 2.53 & 2.01 \\
\hline C267 & $6 \mathrm{wk}$ & $18 \mathrm{~d}$ & 2.64 & 4.20 \\
\hline
\end{tabular}

the eyelid margins themselves were not trimmed, the exposed palpebral eyelid tissue near the margins healed together, as did the sutured palpebral conjunctivae, to form 2 occlusion layers. Upon termination of the initial period of monocular deprivation, the closed eyelids were carefully parted and the palpebral conjunctivae cut open. At the same time, the eyelids of the nondeprived eye were closed following the procedure described above. Because the eyelid margins were not trimmed at the time of eyelid closure, the kitten was able to maintain a palpebral aperture of normal extent throughout the period of visual recovery. This surgical procedure also produced far less corneal irritation than occurs with the conventional procedure for eyelid closure that requires that the eyelid margins be trimmed, with the consequent loss of the sebaceous glands located there.

Following periods of reverse occlusion of various durations, the eyelids of the initially nondeprived eye were opened to allow visual input to both eyes. The rearing histories of all of the animals of this study are listed in Table 1, together with the final acuities achieved by the initially deprived and nondeprived eyes approximately 2 months after binocular visual input was introduced following the period of reverse occlusion.

\section{Behavioral testing}

Visual acuity. Daily measurements of the visual acuity for high-contrast (Michelson contrast close to 1.0) square-wave gratings were made during the period of reverse occlusion and for several weeks afterward by use of a jumping stand and modifications of procedures described earlier (Mitchell et al., 1977b; Mitchell and Timney, 1981). Certain refinements to this procedure, which have been described only in abbreviated form (Mitchell et al., 1984b), are described in more detail here, since they improve significantly both the accuracy and reliability of the acuity measurements. As in earlier studies, kittens were trained to jump toward a large square-wave grating $\left(19.5 \mathrm{~cm}^{2}\right)$ that was placed adjacent to a uniform stimulus of the same size and mean luminance $\left(100 \mathrm{~cd} / \mathrm{m}^{2}\right)$.

The positions of the 2 stimuli were interchanged from right to left in a predetermined pseudo-random order, with correct jumps rewarded with food (pureed chicken liver) and with petting. Incorrect jumps resulted in a denial of these rewards, and the kitten was immediately required to repeat the trial. Whenever possible, training was begun at 

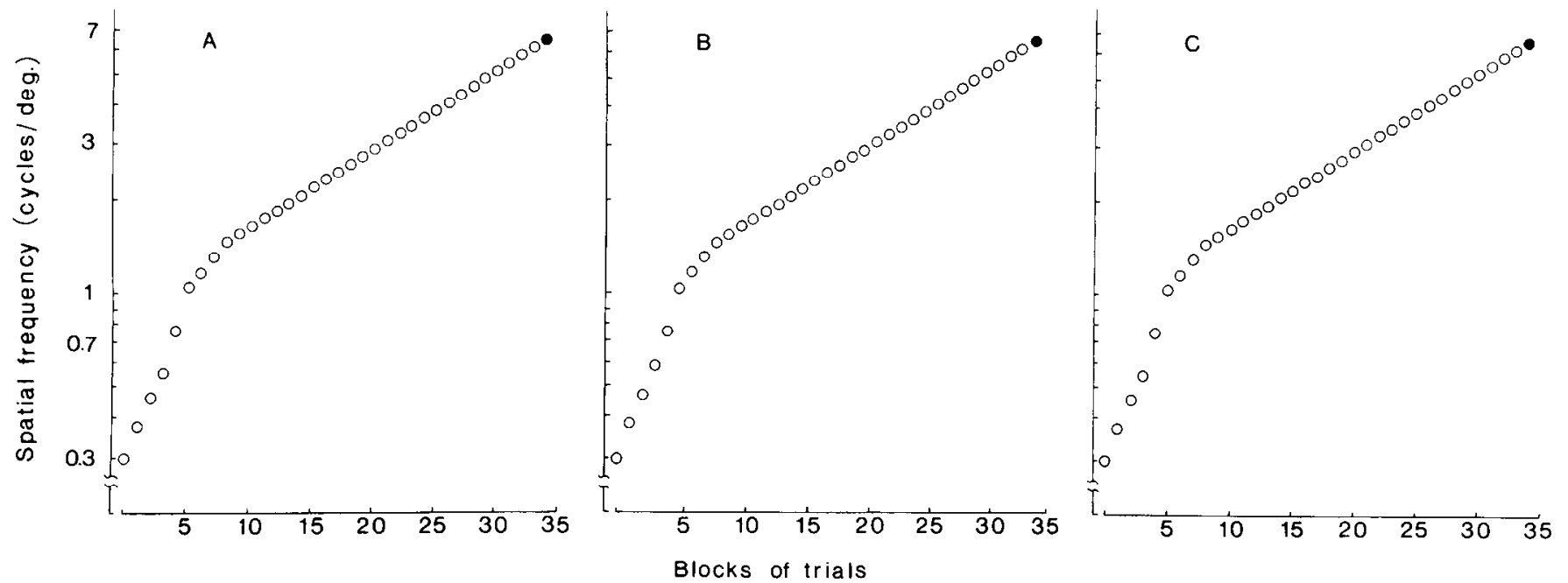

Figure 1. A-C, Performance of a typical cat on 3 consecutive testing sessions. Open symbols represent blocks of trials on which criterion performance (defined in text) was attained, while filled symbols indicate those blocks of trials on which this performance level was not achieved. Note that the spatial frequency of the gratings has been plotted on a logarithmic scale to highlight the fact that above 1 cycle/deg the incremental steps in spatial frequency between successive blocks of trials were both small and approximately equal on such a scale. While still approximately equal, the increments in spatial frequency below 1 cycle/deg were larger than at higher spatial frequencies. The animal's acuity on any session was defined as the highest spatial frequency for which criterion performance could be achieved, in this case $6.1 \mathrm{cycles} / \mathrm{deg}$.

around $30 \mathrm{~d}$ of age using the procedure described earlier (Mitchell et al., 1977b; Giffin and Mitchell, 1978) and was typically completed in 3 to $4 \mathrm{~d}$.

The procedure employed in a typical testing session can be appreciated best by reference to Figure 1, which shows the results from 3 consecutive daily testing sessions on 1 of the animals of this study. Each daily session consisted of many blocks of trials with gratings of progressively higher spatial frequencies that were changed in very small steps between successive blocks of trials until a spatial frequency was reached where the animal was no longer able to maintain a criterion level of performance. The steps were approximately equated on a logarithmic scale, being separated by $1 / \%$ of an octave for gratings having periods of $10-40 \mathrm{~mm}$ (corresponding to spatial frequencies of approximately $0.3-1.2$ cycles/ deg at the largest observation distance of $70 \mathrm{~cm}$ ), $1 / 10$ of an octave for gratings with periods between 3 and $10 \mathrm{~mm}$, and $1 / 12$ of an octave for gratings having periods below $3 \mathrm{~mm}$ (spatial frequencies of $4.1 \mathrm{cycles} /$ deg or higher at $70 \mathrm{~cm}$ ).

Each test session began with gratings of very low spatial frequency, several octaves from their threshold. Usually the animal received only 1 trial at each of the very low spatial frequencies unless an error was made. In such cases additional trials were given until the kitten either made 5 consecutively correct responses or else achieved 7 correct responses in a maximum of 10 trials. Within approximately an octave of the estimated threshold, the minimum number of trials at each spatial frequency (when performance was errorless) was increased to 2 or 3 . This minimum number of trials was increased again to 3 or 5 for the 3 or 4 spatial frequencies closest to threshold. As earlier, following any error the animal had to attain the criterion level of performance described above. Sessions were usually terminated once the animal could no longer achieve criterion within the maximum number of trials (10) permitted at any spatial frequency. During training, or on the rare occasions where it was suspected that the animal's motivation was poor, the procedure was repeated again starting at a spatial frequency an octave lower than that on which the animal had failed. Generally, a test session for a trained animal lasted between 30 and $45 \mathrm{~min}$.

Typically, performance fell from flawless to chance within 2 incremental steps, equivalent to only a small $(\sim 12 \%)$ change in spatial frequency. Because performance declined so rapidly with spatial frequency, the thresholds on any day could be titrated very precisely. As in earlier work, we adopted a very conservative criterion for threshold, namely, the highest spatial frequency for which criterion performance was achieved. At this particular spatial frequency the animal's performance could be at best flawless and at worst $70 \%$ correct in the situation where the maximum 10 trials were required.

Thresholds so defined could not only be specified very accurately, but also with high repeatability. Successive measurements made on a wellmotivated visually mature animal in the same session or on consecutive days were remarkably similar, a point illustrated by the results from the animal shown in Figure 1. Estimates of the acuity of an animal obtained by different experimenters were always very similar, a fact attested to by the regular nature of the data obtained from each animal in this study, which were collected by at least 2 and as many as 4 different people. The steep psychometric functions and the high repeatability of the data from any one animal can be attributed to the use of small increments in spatial frequency between successive blocks of trials, which reduces problems of generalization, and to the use of regular testing schedules.

Acuity measurements were only repeated in a session in situations where the acuity had changed substantially since the previous testing session or when it was suspected that the animal was poorly motivated. In the former situations, the second estimate of acuity was often made using a different observation distance. The acuities estimated in such instances were always closely comparable.

When the initial period of monocular deprivation was short (to 4 or 5 weeks of age), it was not possible to complete training prior to initiation of the period of reverse occlusion. In some cases where the period of reverse occlusion was also brief $(9 \mathrm{~d})$ training was not completed until the end of this period or shortly thereafter. Once trained, the animals were tested every day or second day. Following introduction of binocular visual input, all measurements were made monocularly with a large contact lens occluder inserted in 1 eye. The single exception to this was on the day following termination of reverse occlusion where the vision of the initially deprived eye was sometimes assessed from measurements of acuity made under binocular viewing conditions. This was only done when it was thought that the contact lens occluder might irritate the newly opened eyelids of the initially nondeprived eye. Previously conducted control experiments (Giffin and Mitchell, 1978) had shown that in such cases, where the vision of 1 eye was poor, the visual acuity measured under binocular testing conditions was identical to that of the better eye (in this situation, the initially deprived eye).

Contrast sensitivity. Measurements of contrast sensitivity functions were made on 2 animals by use of procedures that are described in detail elsewhere (Mitchell et al., 1984b). Briefly, the stimuli were presented on a large oscilloscope display (Joyce Electronics, Cambridge) by use of a split-screen display. A stationary vertical sinusoidal grating was presented on one half of the screen and a uniform field of the same average luminance $\left(120 \mathrm{~cd} / \mathrm{m}^{2}\right)$ on the other half. The stimuli were viewed by the cat through a sheet of plate glass that formed the landing surface of the jumping stand. The jumping platform was placed $67 \mathrm{~cm}$ from the stimuli, which, after allowing for an average lean of $10 \mathrm{~cm}$, 

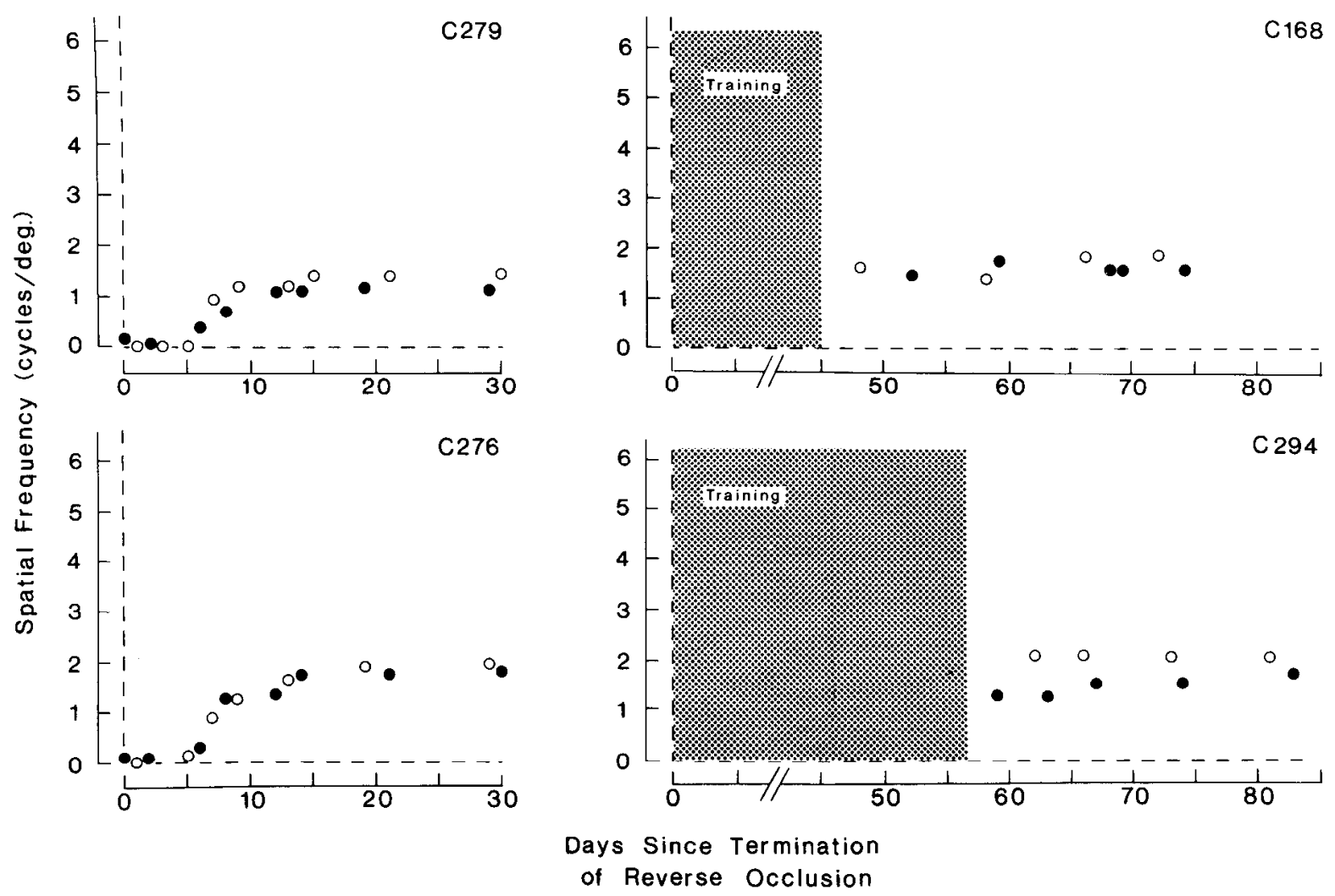

Figure 2. Changes in the visual acuity of the initially deprived (filled symbols) eyes and initially nondeprived (open symbols) eyes of 4 monocularly deprived kittens following termination of $9 \mathrm{~d}$ of reverse occlusion that was initiated when the animals were 4 weeks old. Two animals (C168 and C.294) were not fully trained at the time that reverse occlusion was terminated, and so only the data obtained after training (shaded region) was completed are shown.

meant that the observation distance was $57 \mathrm{~cm}$. At this viewing distance, each stimulus was $20^{\circ}$ high and $14^{\circ}$ wide. Calibration of the oscilloscope display was performed in the manner described by Hess and Baker (1984). Monocular measurements were made of the contrast sensitivity for gratings of only 1 spatial frequency on each daily session, which were repeated for the other eye the next day. Measurements were made first of the contrast sensitivity for gratings of the lowest spatial frequency $(0.25 \mathrm{cycles} / \mathrm{deg})$ and then for gratings of progrcssivcly highcr spatial frequency. The order of testing was then reversed, following which they were reversed again until estimates were obtained for each eye at all spatial frequencies. It required about 4 weeks to generate complete contrast sensitivity functions for both eyes by this procedure.

\section{Results}

\section{Short periods of reverse occlusion}

Results are described first for monocularly deprived animals that were reverse occluded at 4 weeks of age for only $9 \mathrm{~d}$. Binocular visual input was thus introduced when these animals were only just over 5 weeks old, at a time when the visual cortex is most vulnerable to the effects of monocular occlusion (Olson and Freeman, 1980). The changes in the visual acuity of both eyes of 4 such kittens following introduction of binocular visual input are shown in Figure 2. Two of these animals (C168 and C294) were not trained at the time that reverse occlusion was terminated, and so only the data obtained from these animals after 2 months of binocular vision are shown. Although training was begun early on the other 2 kittens (C276 and C279), the nature of the early visual experience of these animals may have prevented training from being fully complete by the end of the period of reverse occlusion since for much of this period the vision of the animal was very poor. During the $9 \mathrm{~d}$ of reverse occlusion the animals recovered only rudimentary vision, estimated to be only 0.1 cycles/deg at the end of $9 \mathrm{~d}$. As in later figures, the visual acuity attained by the initially deprived eye during the period of occlusion is plotted on the extreme left (at zero days on the abscissa). The vision of this eye remained static for about $5 \mathrm{~d}$, after which it began to show slight improvement. -Following 1 to 2 months of binocular visual exposure, the acuity of the initially deprived eye of all 4 animals was less than 2 cycles/deg. This value is only $1 / 4$ to $1 / 3$ of the acuity $(6.4-8.6$ cycles/ deg) of normal animals of comparable age measured in this laboratory under similar conditions during the last 10 years.

Possibly even more remarkable than the very limited recovery of vision exhibited by the initially deprived eye during the period of binocular visual exposure was the similar lack of improvement of the vision of the other eye, which rose from blindness to only 1.5-2.1 cycles/deg during this period. Hence despite the early introduction of binocular visual input, these kittens developed very severe bilateral amblyopia. A similar result was also observed in another kitten (C170) that was also monocularly deprived until 4 weeks, then reverse occluded for a somewhat longer period ( 2 weeks). Subsequently, following a month of binocular visual experience, the visual acuity of the initially nondeprived eye was only $2.3 \mathrm{cycles} / \mathrm{deg}$, while that of the initially deprived eye had attained a level of only 0.6 cycles $/ \mathrm{deg}$.

A similar final outcome was also observed in the 4 kittens monocularly deprived until 5 weeks of age, then reverse occluded for either 9 or $18 \mathrm{~d}$ (see Fig. 3). As might have been anticipated, the improvement in the acuity of the initially deprived eye during $18 \mathrm{~d}$ of reverse occlusion $(\mathrm{C} 155$ and $\mathrm{C} 164)$ 
18 days $R O$

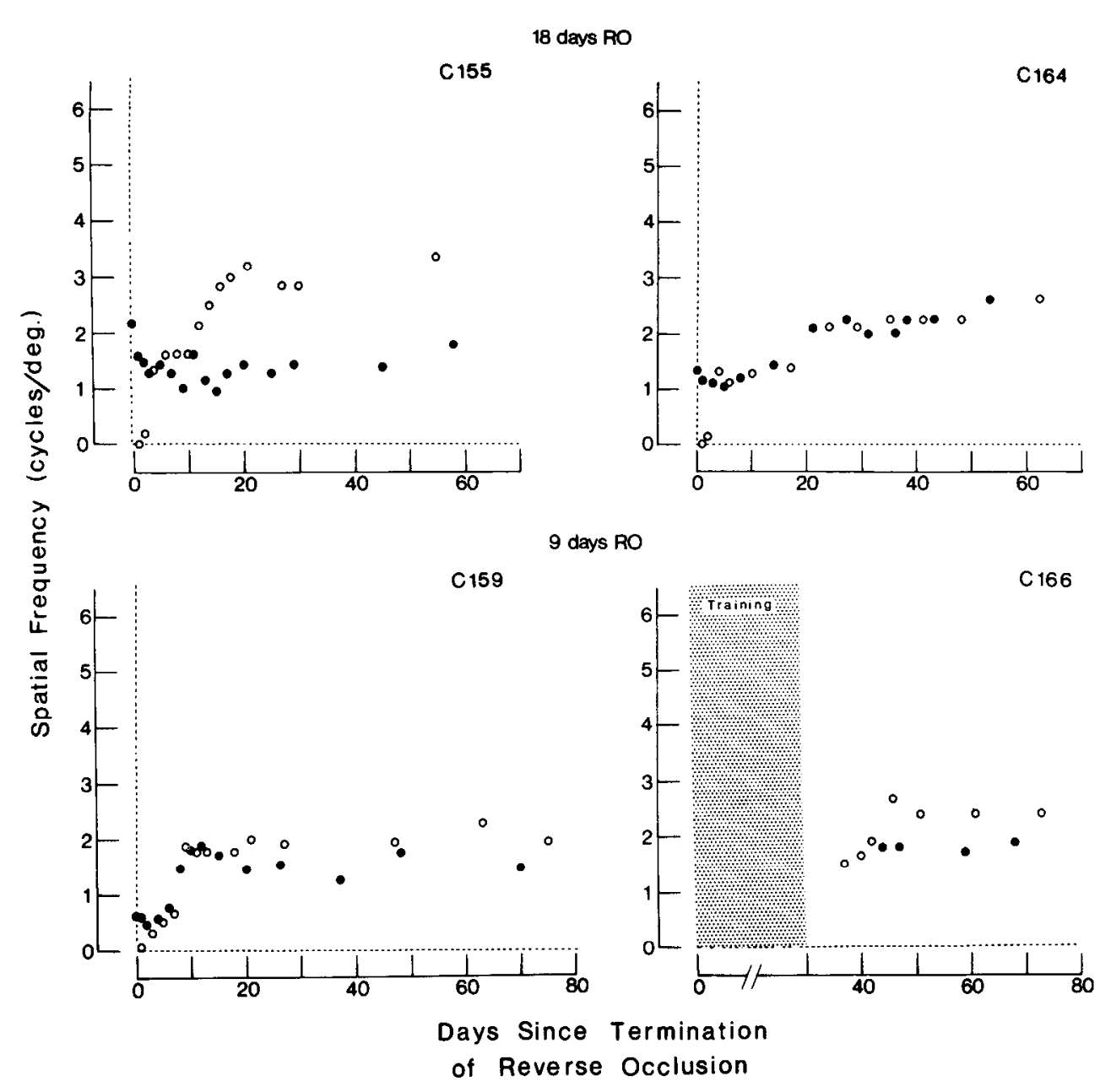

Figure 3. Changes in the visual acuity of the initially deprived (filled symbols) and nondeprived eyes (open symbols) of 4 kittens that were monocularly deprived until 5 weeks of age and then reverse occluded for either $9 \mathrm{~d}(\mathrm{C} 159$ and $\mathrm{C} 166)$ or $18 \mathrm{~d}(\mathrm{C} 155$ and $\mathrm{C} 164)$. Only the final acuities are shown for 1 animal (C166) that was not trained at the time that reverse occlusion was terminated. was slightly greater than what occurred in only $9 \mathrm{~d}$ (C159 and C166). However, in both situations the vision of this eye either dropped (C155) or else improved only very slightly during the subsequent 2 months of binocular visual experience. As a result, after more than 2 months of binocular vision, the acuity of the

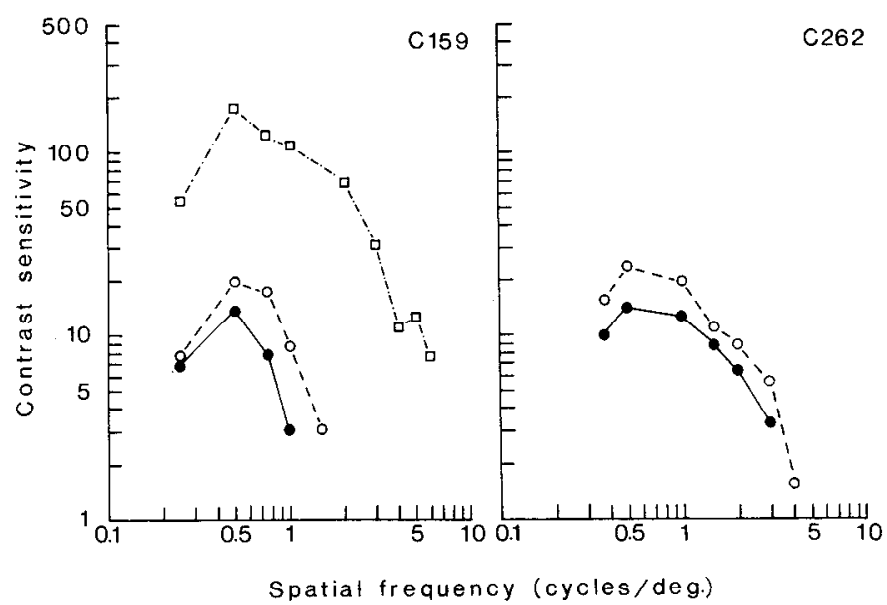

Figure 4. Contrast sensitivity functions for the initially deprived (filled symbols) and nondeprived eyes (open symbols) of 2 monocularly deprived animals that were reverse occluded for $18 \mathrm{~d}$ at either 5 weeks (C159) or 6 weeks (C262) of age and then allowed 3 months of binocular visual experience. For comparison, the data from a typical normal animal of the same age is depicted by open squares in the left-hand panel. initially deprived eye was less than $1 / 4$ of the optimum values achieved by normal animals of comparable age. During the period of binocular exposure the acuity of the initially nondeprived eye improved to levels that were slightly higher than same level in the fourth animal (C164). The difference between the acuities of the 2 eyes was quite substantial for 1 animal (C155), but even so, the acuity of the better (initially nondeprived) eye remained at levels that were less than $1 / 2$ of normal values. Ovcrall, there was a tendency for the animals that were reverse occluded for $18 \mathrm{~d}$ to attain acuities that were slightly higher in their initially nondeprived eye (range, 2.6-3.4 cycles/ deg) than did those animals that were reverse occluded for only $9 \mathrm{~d}$ (range, $2.0-2.5$ cycles/deg).

The substantial bilateral amblyopia that was exhibited eventually by animals that were reverse occluded early and for short periods of time was even more evident in the measurements of the contrast sensitivity functions for the 2 eyes. Results for such an animal (C159 of Fig. 3) are shown in the left-hand panel of animal of similar age. The contrast sensitivities of the 2 eyes were approximately equal and very poor at all spatial frequencies. At the peak of the contrast sensitivity function $(0.5$ cycles/ $\mathrm{deg}$ ), the contrast sensitivity of $\mathrm{C} 159$ was reduced by a factor of nearly 10 from that of the normal animal. This is substantially greater than the observed 3- to 4-fold reduction in grating acuity.

Figure 5 displays results from 2 representative kittens from those of the initially deprived eye in 3 cases but to only the Figure 4 together with the monocular results from a normal 


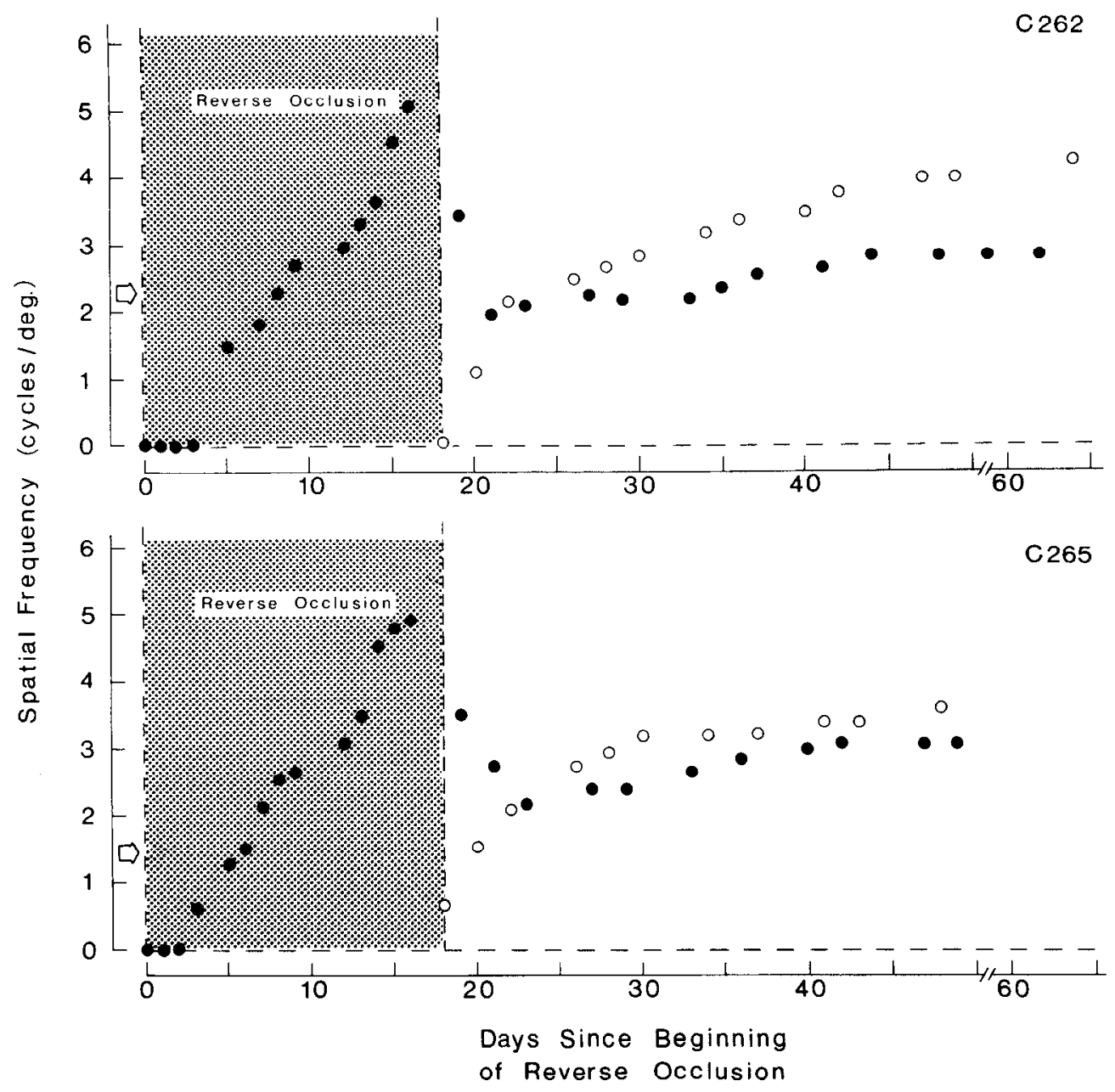

Figure 5. Changes in the visual acuity of the deprived eye of 2 monocularly deprived kittens during an $18 \mathrm{~d}$ period of reverse occlusion (shaded region), together with the changes that occurred afterwards to the acuity of this eye, as well as to the acuity of the initially nondeprived eye (open symbols) upon introduction of binocular visual experience. The period of reverse occlusion was imposed when the kittens were 6 weeks old. The open arrows indicate the acuity of the initially nondeprived eye at the end of this initial 6 week period of monocular deprivation. a group of 4 animals that were monocularly deprived until 6 weeks of age and then reverse occluded for $18 \mathrm{~d}$. During the short period of reverse occlusion the vision of the initially deprived eye recovered rapidly and substantially from blindness to an acuity of about 5 cycles/deg. Despite the substantial recovery of vision by the initially deprived eye during the period of reverse occlusion, the acuity of this eye deteriorated rapidly afterwards to about 2 cycles/deg in only 3-5 days. The vision of this eye recovered a little in the next 2 months, but only to about 3 cycles/deg, a level well below that which it had reached during reverse occlusion. Concurrent with the rapid decline in the vision of this eye upon introduction of binocular vision, the vision of the initially nondeprived eye improved gradually from the levels to which it had been reduced during reverse occlusion. For 2 of the 4 kittens reared in this condition, the initially nondeprived eye was rendered blind by the period of reverse occlusion, but for the other 2 kittens, the vision of this eye was not reduced as severely $(0.5$ and 1.0 cycles/deg). During the first 2 weeks of binocular exposure, the vision of this eye improved rapidly to exceed the acuity it had achieved earlier during the initial period of monocular occlusion (indicated by open arrows), as well as the acuity of the other eye. The rate of improvement of vision slowed markedly thereafter, so that after 2 months the acuity of this eye was less than $1 / 2$ of the acuity of normal animals. Comparable final results were also observed in the other 2 animals (C267 and C269) of this group (Table 1).
Contrast sensitivity functions were determined for both eyes of one of the kittens of this group (C262) several months after the data shown in Figure 5 had been collected. 'The results of these measurements, which are displayed in Figure 4 (right panel), indicate that the contrast sensitivity of both eyes was about an order of magnitude below normal at all spatial frequencies.

\section{Long periods of reverse occlusion}

The consequences for the vision of the 2 eyes of substantially longer periods of reverse occlusion, 9 weeks or more, was studied in 7 kittens. The changes in the vision of both eyes during the period of binocular vision for 2 of these animals that were reverse occluded for 9 weeks at either 4 (C181) or 5 weeks (C142) of age are shown in Figure 6. During the long period of reverse occlusion the vision of the initially deprived eye of both animals recovered to levels that were within the range of values encountered in normal animals. Despite the fact that the animals were 13 to 14 weeks old when reverse occlusion was terminated, the vision of this eye dropped dramatically and rapidly within days of restoring visual input to the initially nondeprived eye. During the first $12 \mathrm{~d}$ of binocular visual experience, the vision of the initially deprived eye dropped to levels (below 2 cycles/ deg) that were less than $1 / 3$ of the values it had attained during reverse occlusion. Although the vision of this eye improved slightly in the weeks that followed, it remained below 2 cycles/ deg.

While the changes in the vision of the initially deprived eye 
Figure 6. Changes in the visual acuities of the initially deprived (filled symbols) and nondeprived eyes (open symbols) of 2 kittens following termination of 9 weeks of reverse occlusion that was imposed at either $4(\mathrm{C} 181)$ or $5(\mathrm{C} 142)$ weeks of age.

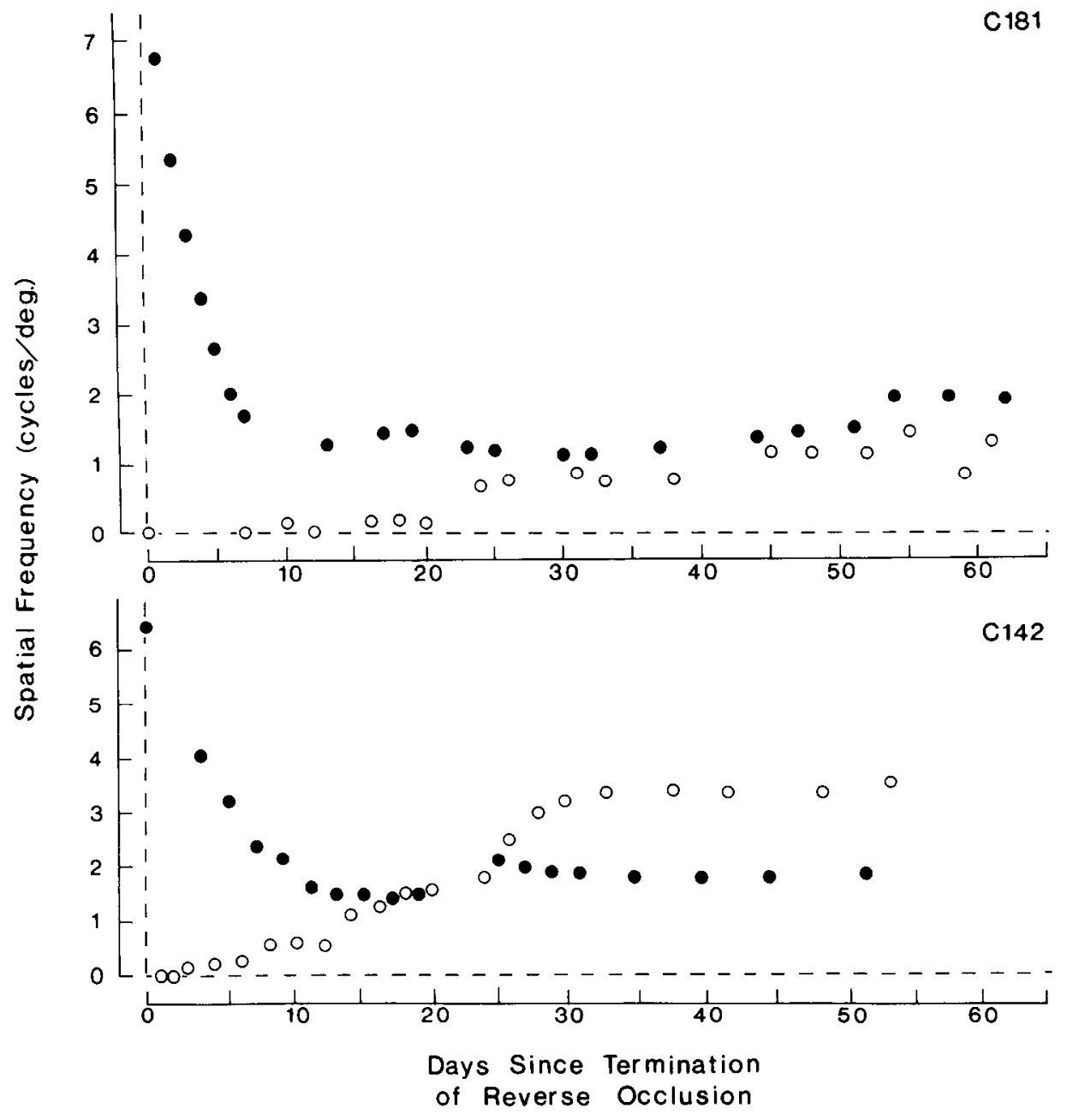

kitten was 16 weeks old at this point, the acuity of the initially deprived eye dropped precipitously during the first 2 weeks of binocular visual exposure. Interestingly, this fall in acuity did not begin immediately; the acuity of the initially deprived eye rèmained at 6.1 cycles/deg during the first $5 \mathrm{~d}$ of binocular visual exposure, but in the course of the next 10 days it fell to only $2.6 \mathrm{cycles} / \mathrm{deg}$, where it remained throughout the period of testing. Throughout this period of rapid change in the vision of the initially deprived eye, the other eye remained blind. The first signs of vision in this eye were evident only after 2 weeks of binocular visual exposure, after which it improved very gradually and to only a very limited extent. At the conclusion of testing, when the animal was 22 weeks old, this eye had attained an acuity of only 1.2 cycles $/ \mathrm{deg}$.

Results from a representative kitten $(\mathrm{C} 157)$ from a group of 3 kittens monocularly deprived until 5 weeks of age and then reverse occluded for 12 weeks are shown in Figure 8. At the time that reverse occlusion was terminated, the animals were 17 weeks old, an age that corresponds to the latter stages of the sensitive period as originally defined (Hubel and Wiesel, 1970). As with the cat represented in Figure 7, the acuity of the initially deprived eye of all 3 of these animals had recovered to 6 cycles/ deg or better by the end of the 12 week period of reverse occlusion. The vision of the initially deprived eye of $\mathrm{C157}$ remained at this level during the first $3 \mathrm{~d}$ of binocular vision but fell rapidly afterwards to about 2.3 cycles/deg within a week, a 


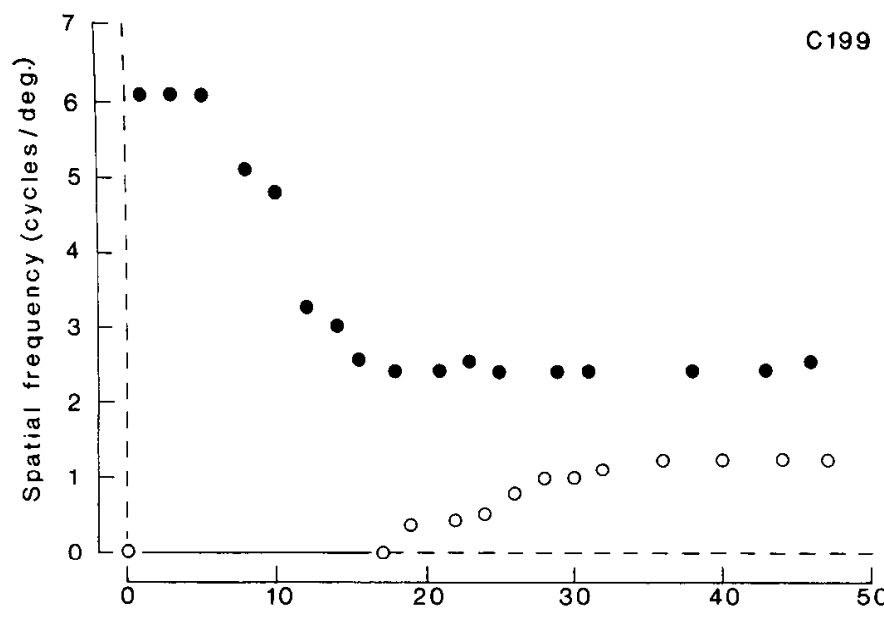

Days since termination of reverse occlusion

Figure 7. Changes in the visual acuities of the initially deprived (filled symbols) and nondeprived eye (open symbols) of a monocularly deprived kitten (C199) following termination of 12 weeks of reverse occlusion that had been imposed when the kitten was only 4 weeks old. During the period indicated by the continuous horizontal line that joins the open circles, the animal appeared blind when using its initially nondeprived eye.

level from which it never recovered. The initially nondeprived eye remained blind during the first $11 \mathrm{~d}$ of binocular exposure but improved slowly thereafter. The vision of this eye never reached that of the other eye and eventually attained an acuity of only 1.2 cycles/deg.

\section{Discussion}

Paradoxically, both of the 2 extreme regimens of reverse occlusion examined here eventually lead to the development of severe bilateral amblyopia. In every case, reverse occlusion was initiated at only 4,5 , or 6 weeks of age, when the visual cortex is most susceptible to environmental modification. At one extreme, where the period of reverse occlusion was short but physiologically optimal (in the sense that it was sufficiently long to allow for a complete shift of ocular dominance in the visual cortex), binocular visual experience was introduced when the animals were still very young. Despite this, there was very little functional improvement in the acuity of the initially deprived eye beyond the level it had reached during the short period of reverse occlusion. Even more surprising was the lack of substantial improvement of the vision of the initially nondeprived eye following termination of reverse occlusion. At the other extreme, reverse occlusion was continued for several months and therefore terminated very late in relation to the durations of known critical periods in the visual pathways. During this long period of reverse occlusion the vision of the initially deprived eye improved to levels that were within the range of values encountered among normal animals of comparable age. Surprisingly, however, the vision of this eye was not maintained once reverse occlusion was terminated. Moreover, the vision of the other (initially nondeprived) eye was permanently compromised by the long period of reverse occlusion. Thus, like the animals reverse occluded for short periods, the end result was severe bilateral amblyopia.

The present results both confirm and extend the findings of an earlier study of the permanence of the visual recovery promoted by reverse occlusion (Mitchell et al., 1984b). Several kittens in the earlier study were initially monocularly deprived for periods of time identical to those employed here; however, the length of the period of reverse occlusion that followed fell midway between the 2 extreme conditions employed in the present investigation. Following termination of reverse occlusion of such intermediate duration, there was a striking reciprocal change in the visual acuity of the 2 eyes: The vision of the initially deprived eye fell from the substantial levels it had reached during reverse occlusion, while concurrently the vision of the other (initially nondeprived) eye improved to levels that were close to (but still below) normal values. The most noteworthy feature of the final outcome from animals subjected to those particular regimens of reverse occlusion was the severity of the amblyopia in the initially deprived eye. The vision of the initially nondeprived eye was compromised to a much lesser extent.

When this earlier data is combined with that of the present study an interesting relationship emerges between the duration of reverse occlusion and the visual acuity that the 2 eyes eventually attain after several months of binocular visual exposure. These data are summarized in Figure 9, which shows a plot of the mean final acuity of the 2 eyes of each group of animals as a function of the length of reverse occlusion. The top panel displays the mean acuities from the kittens of the present study that were monocularly deprived to 4 weeks of age, together with

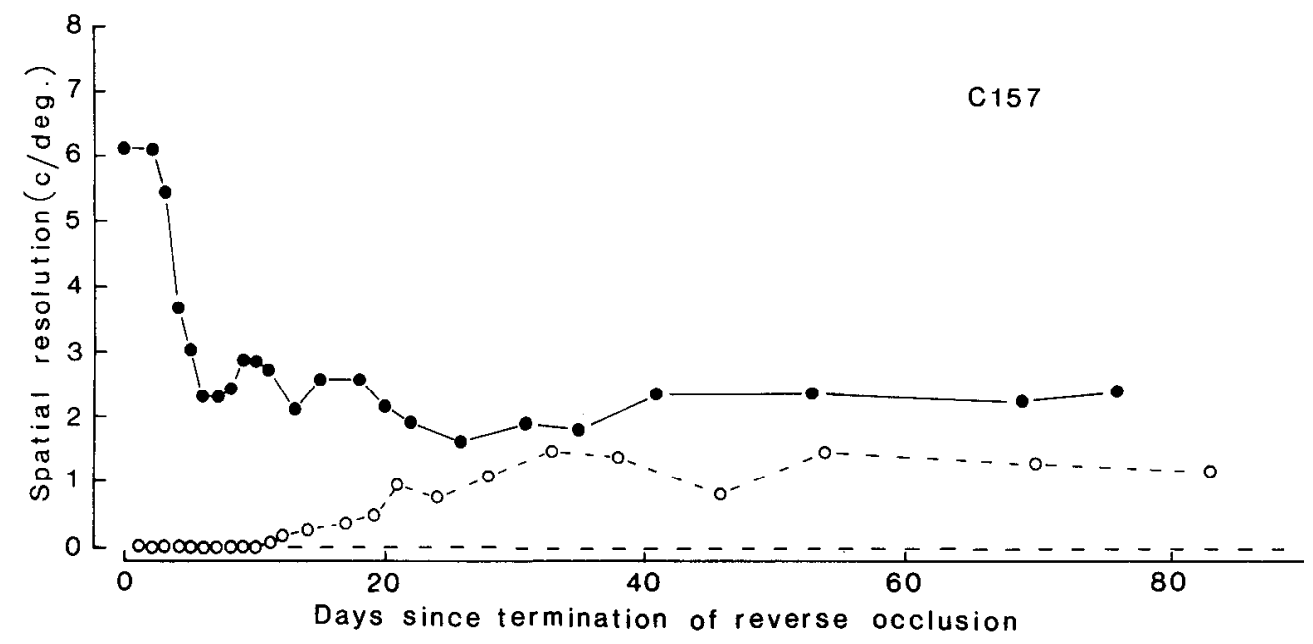

Figure 8. Changes in the visual acuity of the initially deprived (filled symbols) and nondeprived eye (open symbols) of a monocularly deprived kitten (C157) following termination of 12 weeks of reverse occlusion that had been imposed when the animal was 5 weeks old. 


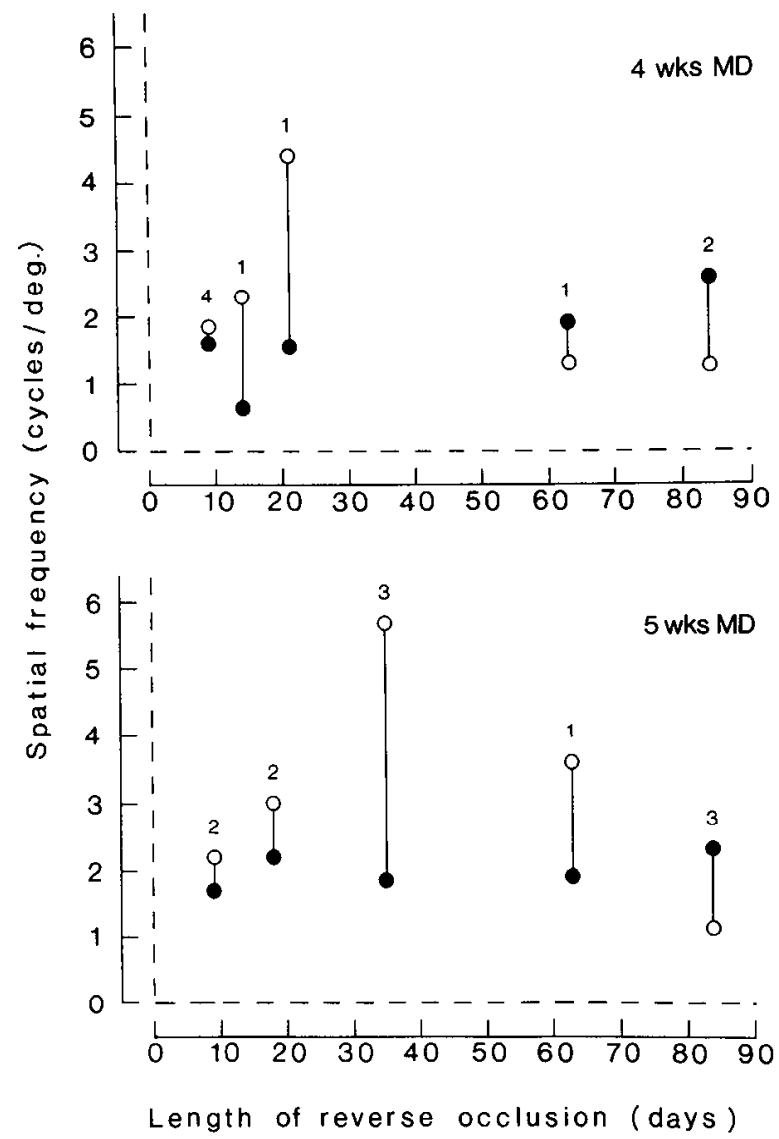

Figure 9. Summary of the mean final acuities achieved several months after termination of different periods of reverse occlusion imposed on kittens that had been monocularly deprived until either 4 weeks (top panel) or 5 weeks (bottom panel) of age. Connected filled and open symbols depict the mean final acuities of, respectively, the initially deprived and nondeprived eyes of groups of kittens that were reverse occluded for the periods indicated on the abscissa. The numbers above each connected set of symbols represent the number of animals in each group. Included in this figure are data from 2 animals from an earlier study (Mitchell et al., 1984b), as well as 2 additional cats from a study in progress. For comparison, the acuity of normal cats is betwen 6.4 and $8.6 \mathrm{cycles} / \mathrm{deg}$.

the results from 1 kitten $(\mathrm{C} 158)$ from the earlier study (Mitchell et al., 1984b) that was reverse occluded for $21 \mathrm{~d}$, beginning at 4 weeks of age. Data for kittens monocularly deprived to 5 weeks are shown in the lower panel. Included in the lower panel are results from 3 kittens (including C141 of Mitchell et al., 1984b) that were reverse occluded for $35 \mathrm{~d}$. For both sets of data it is apparent that after several months of binocular exposure the vision of the initially deprived eye drops to extremely low levels, irrespective of the length of reverse occlusion. On the other hand, the relationship between the length of reverse occlusion and the acuity eventually attained by the initially nondeprived eye is more complex. With a period of reverse occlusion that is either very short (9-18 d) or else very long (9-12 weeks), the initially nondeprived eye became severely amblyopic-in some cases even more so than the initially deprived cyc! However, following periods of reverse occlusion of intermediate duration the initially nondeprived eye recovers good (but still subnormal) acuity. At this stage we can offer no explanation for the variety of effects observed in the 2 eyes following termination of the various regimens of reverse occlusion examined to date. It is cven possible that these effects may reflect a number of different underlying mechanisms. However, it is possible to eliminate a number of possible candidates. These are discussed individually below, together with comments upon other likely mechanisms.

In discussion of the earlier findings (Mitchell et al., 1984b), it was pointed out that the visual changes that followed introduction of binocular visual input after periods of reverse occlusion were very different from those that occur when a period of binocular exposure follows a single period of monocular occlusion imposed near birth. In the latter circumstance, the recovery of vision that occurs in the deprived eye is not accompanied by any loss of acuity in the nondeprived eye (Mitchell et al., 1977a; Giffin and Mitchell, 1978). On the other hand, following termination of all but short periods of reverse occlusion, the improvement in the vision of the initially nondeprived eye was accompanied by a rapid loss of acuity of the other eye. The substantial difference between these outcomes is even more remarkable in view of the physiological status of the visual cortex at the time that binocular visual input is introduced in the 2 situations. In both cases the ocular dominance of cortical cells is markedly biased in favor of 1 eye, the nondeprived eye after a single period of monocular occlusion and the initially deprived eye at the end of a period of reverse occlusion of sufficient duration (Movshon, 1976). The fact that the conscquences for the vision of the 2 eyes during a subsequent period of binocular exposure are so different suggests that the mechanisms responsible for the physiological changes promoted by reverse occlusion must be in some way different from those that take place during the initial period of monocular deprivation.

\section{Shifts of cortical ocular dominance}

It is not unreasonable to propose that the changes in vision that follow termination of reverse occlusion reflect nothing more than the shifts of ocular dominance of cells that occur in cortical area 17 during the period of binocular exposure. Explanations for the different outcomes that follow various regimens of reverse occlusion could then be sought in terms of differences between their effects on cortical ocular dominance. Although electrophysiological investigations have been made on only a few animals, it appcars that the visual effects that are summarized in Figure 9 cannot be explained simply in terms of shifts of cortical ocular dominance. Preliminary investigations of cortical ocular dominance have been made on 4 kittens a month after termination of an $18 \mathrm{~d}$ period of reverse occlusion imposed at 5 weeks of age (Murphy and Mitchell, 1986). In contrast to the highly biased and monocular distribution of ocular dominance that is observed at the end of $18 \mathrm{~d}$ or more of reverse occlusion (Movshon, 1976), the distribution observed following a month of binocular exposure was highly binocular with neither eye dominating more cells than the other. Furthermore, the receptive fields appeared qualitatively normal. Clearly these qualitative electrophysiological findings provide no ready explanation for the poor visual acuity of the 2 eyes in this situation. A similar conclusion can be drawn from the electrophysiological findings from an animal that was reverse occluded for a longer period (6 weeks) at 6 weeks of age (Mitchell et al., 1984a). Although the distribution of cortical ocular dominance recorded in this animal after several months of binocular exposure was quite different from that reported from animals recorded immediately after a comparable period of reverse occlusion (Movshon, 1976), the difference between the proportion of cells driv- 
en by the 2 eyes was small in comparison to the profound differences between the acuities of the 2 eyes. However, as suggested earlier (Mitchell et al., 1977a; Blakemore and Eggers, 1978; Giffin and Mitchell, 1978), the acuity of an eye may be related more closely to the spatial resolution of the most sensitive cortical cells driven by that eye than to the absolute number or proportion of cells which that eye dominates. Consequently, it might be fruitful to examine in a quantitative fashion the spatial resolution of cells driven by the 2 eyes of animals reared with the various regimens of reverse occlusion examined here.

\section{Strabismus}

Unless the visual axes of the 2 eyes remained aligned during the period of binocular exposure, the visual input to the 2 eyes would not be concordant. It was suggested earlier (Mitchell et al., 1984b) that the rapid loss of acuity of the initially deprived eye during the first few days of binocular exposure may represent the sudden onset of strabismic amblyopia in this eye arising as a consequence of such misalignment of the visual axes. A number of powerful arguments were advanced then against this explanation, including the fact that a similar phenomenon does not occur during binocular recovery following early monocular deprivation, when a strabismus is just as likely to be present. In addition, the time course of the changes in visual acuity following termination of reverse occlusion are far more rapid than the rather gradual onset of amblyopia following imposition of a surgically induced strabismus (Mitchell et al., 1984c). The data from the present study provides additional arguments against this idea. As mentioned earlier, the ocular dominance distributions from animals that were reverse occluded for short periods of time were highly binocular, a result inconistent with the presence of a strabismus (Van Sluyters and Levitt, 1980; Levitt and Van Sluyters, 1982). In addition, the ocular dominance distribution from the animal described very briefly earlier (Mitchell et al., 1984a) exhibited a degree of binocularity that was also inconsistent with strabismus. Finally, the loss of acuity in the animals reverse occluded for long periods of time is also inconsistent with such an explanation, since strabismic amblyopia has not been observed to develop in kittens on which a strabismus was induced at the advanced age at which binocular visual input was introduced to those animals. Although these lines of evidence do not rule out altogether the possibility that the presence of a strabismus may contribute to the behavioral findings, they suggest very strongly that the contribution is a minor one.

\section{Effects on layer IV in the visual cortex}

Mitchell et al. (1984b) previously proposed that the difference between the effects observed when binocular visual input was introduced after a period of early monocular deprivation, as opposed to those that follow reverse occlusion, may be due, in part, to a differential effect of the 2 deprivation regimens on the ocular dominance of cells in layer IV of the visual cortex. During normal development, the segregation of geniculocortical afferents into ocular dominance columns in layer IV is complete by about 6 weeks of age (LeVay et al., 1978). Many of the cats of the earlier study were reverse occluded after 6 weeks of age, at which time changes of ocular dominance in layer IV would be minimal. The changes in acuity that occurred during reverse occlusion of those animals must have largely represented ana- tomical and physiological events in layers of the cortex other than layer IV. By contrast, a period of monocular deprivation imposed near birth will exert strong effects on layer IV, as well as on other layers. Consequently, the different effects on visual acuity observed during subsequent binocular exposure in these 2 situations may simply reflect the differential effects on layer IV. However, in the present study, most animals were reverse occluded at an age when the process of segregation is normally still in progress. Nevertheless, the final acuity of the initially deprived eye in all the kittens of the present study was extremely poor. Furthermore, in the situation where reverse occlusion was initiated early (at 4 weeks) and extended for long periods, the effects on ocular dominance in layer IV during this period might be expected to be substantial. However, the acuity of the initially deprived eye of these animals declined just as rapidly and to the same degree as in any of the other conditions studied (Fig. 9). Therefore, it does not seem likely that the poor acuity of the initially deprived eye can be related in any simple way to the onset of the period of reverse occlusion in relation to the normal time course of segregation of the geniculocortical afferents in layer IV.

However, it is still possible that certain changes in layer IV may be an important contributing factor to the severe bilateral amblyopia observed among the kittens in this study. One possible explanation for this surprising finding is that the geniculocortical afferents from both eyes may have expanded axonal arborization fields. Previous work in monkeys has shown that during reverse occlusion the projections to layer IV from the initially deprived eye expand (LeVay et al., 1980; Swindale et al., 1981). However, until recently there was little information concerning the concurrent changes that might occur to the afferents from the initially nondeprived eye during this period. Recently, measurements of LGN cell cross-sectional areas following a short period of reverse occlusion in monkeys indicate that cells in laminae that receive input from the initially nondeprived eye remain hypertrophied during reverse occlusion (Sloper et al., 1984), suggesting that they may be supporting an enlarged geniculocortical arborization. Such overly exuberant projections to the visual cortex from both eyes may lead both to changes in receptive field properties and to smearing of the retinotopic map on the cortex. Either of these changes could account for the poor acuity observed in the cats of this study. These and other possible explanations are currently under investigation. It is also possible that similar anatomical changes may underlie some of the perceptual distortions experienced by human amblyopes, which in recent years have been attributed to as yet unspecified scrambling of the neural signals from the amblyopic eye (e.g., Hess et al., 1978; Bedell and Flom, 1981).

The poor eventual outcome from the 2 regimens of reverse occlusion explored in this investigation raise serious questions concerning the usefulness of full-time reverse occlusion as a means for promoting recovery from the effects of early monocular deprivation. Indeed, this view reinforces a similar conclusion that could be drawn from an earlier investigation (Mitchell et al., 1984b). In only a very limited set of conditions in that earlier study did the vision of the initially deprived eye eventually recover to levels close to normal. In view of the generally poor outcome from the majority of full-time reverse occlusion regimens, we have begun to explore the consequences of certain regimens of part-time reverse occlusion, where the initially deprived eye is reverse occluded for only part of each day in order 
to allow a daily period of binocular visual exposure. Preliminary findings suggest that certain regimens of part-time reverse occlusion permit the eventual recovery of normal visual acuity in both eyes (Mitchell et al., 1986).

\section{References}

Bedell, H. E., and M. C. Flom (1981) Monocular spatial distortion in strabismic amblyopia. Invest. Ophthalmol. Vis. Sci. 20: 263-268.

Blakemore, C., and H. M. Eggers (1978) Animal models for human visual development. In Frontiers of Visual Science, S. J. Cool and E. L. Smith, eds., pp. 651-659, Springer-Verlag, New York.

Blakemore, C., L. J. Garey, and F. Vital-Durand (1978) The physiological effects of monocular deprivation and their reversal in the monkey's visual cortex. J. Physiol. (Lond.) 282: 223-262.

Blakemore, C., F. Vital-Durand, and L. J. Garey (1981) Recovery from monocular deprivation in the monkey. I. Recovery of physiological effects in the visual cortex. Proc. R. Soc. London [Biol.] 213: 399-423.

Cynader, M., B. N. Timncy, and D. E. Mitchell (1980) Period of susceptibility of kitten visual cortex to the effects of monocular deprivation extends beyond six months of age. Brain Res. 191: 545550.

Giffin, F., and D. E. Mitchell (1978) The rate of recovery of vision after early monocular deprivation in kittens. J. Physiol. (Lond.) 274: 511-537.

Guillery, R. W. (1972) Binocular competition in the control of geniculate cell growth. J. Comp. Neurol. 144: 117-130.

Guillery, R. W., and D. J. Stelzner (1970) The differential effects of unilateral lid closure upon the monocular and binocular segments of the dorsal lateral geniculate nucleus in the cat. J. Comp. Neurol. 139: 413-422.

Hess, R. F., and C. L. Baker (1984) Human pattern-evoked electroretinogram. J. Neurophysiol. 51: 939-951.

Hess, R. F., F. W. Campbell, and T. Greenhalgh (1978) On the nature of the neural abnormality in amblyopia: Neural aberration and neural sensitivity loss. Pfluegers Arch. 377: 201-207.

Hubel, D. H., and T. N. Wiesel (1970) The period of susceptibility to the physiological effects of unilateral eye closure in kittens. J. Physiol. (Lond.) 206: 419-436.

Hubel, D. H., T. N. Wiesel, and S. LeVay (1977) Plasticity of ocular dominance columns in monkey striate cortex. Phil. Trans. R. Soc. London [Biol.] 278: 377-409.

Jones, K. R., P. D. Spear, and L. Tong (1984) Critical periods for effects of monocular deprivation: Differences between striate and extrastriate cortex. J. Neurosci. 4: 2543-2552.

LeVay, S., M. P. Stryker, and C. J. Shatz (1978) Ocular dominance columns and their development in layer IV of the cat's visual cortex: A quantitative study. J. Comp. Neurol. 179: 223-244.

LeVay, S., T. N. Wiesel, and D. H. Hubel (1980) The development of ocular dominance columns in normal and visually deprived monkeys. J. Comp. Neurol. 191: 1-51.
Levitt, F. B., and R. C. Van Sluyters (1982) The sensitive period for strabismus in the kitten. Dev. Brain Res. 3: 323-327.

Mitchell, D. E., and B. N. Timney (1981) Behavioral measurement of normal and abnormal development of vision in kittens. In Analysis of Visual Behavior, D. J. Ingle, M. A. Goodale, and R. J. Mansfield, eds., pp. 483-523, MIT Press, Cambridge, MA.

Mitchell, D. E., and B. N. Timney (1984) Postnatal development of function in the mammalian visual system. In Handbook of Physiology-Nervous System, Vol. 3, Sensory Processes, Part 1, I. DarianSmith, ed., pp. 507-555, American Physiological Society, Bethesda, MD.

Mitchell, D. E., M. Cynader, and J. A. Movshon (1977a) Recovery from the effects of monocular deprivation in kittens. J. Comp. Neurol. 176: 53-64.

Mitchell, D. E., F. Giffin, and B. N. Timney (1977b) A behavioral technique for the rapid assessment of the visual capabilities of kittens. Perception 6: 181-193.

Mitchell, D. E., K. M. Murphy, and M. G. Kaye (1984a) Labile nature of the visual recovery promoted by reverse occlusion in monocularly deprived kittens. Proc. Natl. Acad. Sci. USA 81: 286-288.

Mitchell, D. E., K. M. Murphy, and M. G. Kaye (1984b) The permanence of the visual recovery that follows reverse occlusion in monocularly deprived kittens. Invest. Ophthal. Vis. Sci. 25: 908-917.

Mitchell, D. E., M. Ruck, M. G. Kaye, and S. Kirby (1984c) Immediate and long-term effects on visual acuity of surgically induced strabismus in kittens. Exp. Brain Res. 55: 420-430.

Mitchell, D. E., K. M. Murphy, H. A. Dzioba, and J. A. Horne (1986) Optimization of visual recovery from early monocular deprivation in kittens: Implications for occlusion therapy in the treatment of amblyopia. Clin. Vis. Sci. $1: 173-177$.

Movshon, J. A. (1976) Reversal of the physiological effects of monocular deprivation in the kitten's visual cortex. J. Physiol. (Lond.) 261: $125-175$.

Movshon, J. A., and R. C. Van Sluyters (1981) Visual neuronal development. Annu. Rev. Psychol. 32: 477-522.

Murphy, K. M., and D. E. Mitchell (1986) Bilateral amblyopia following a short period of reverse occlusion. Nature 323: 536-538.

Olson, C. R., and R. D. Freeman (1980) Profile of the sensitive period for monocular deprivation in kittens. Exp. Brain Res. 39: 17-21.

Sloper, J. J., M. P. Headon, and T. P. S. Powell (1984) Simultaneous hypertrophy of cells related to each eye in the lateral geniculate nucleus of the infant monkey following short-term reverse suture. Dev. Brain Res. 15: 295-297.

Swindale, N. V., F. Vital-Durand, and C. Blakemore (1981) Recovery from monocular deprivation in the monkey. III. Reversal of anatomical effects in the visual cortex. Proc. R. Soc. London [Biol.] 213: 435-450.

Van Sluyters R. C., and F. I evitt (1980) Fxperimental strabismus in the kitten. J. Neurophysiol. 43: 686-699.

Wiesel, T. N., and D. H. Hubel (1963) Single-cell responses in the striate cortex of kittens deprived of vision in one eye. J. Neurophysiol. 26: $1003-1017$ 